OPEN ACCESS

Edited by: loan Opris,

Leonard M. Miller School of Medicine,

United States

Reviewed by:

Filippo Brighina,

University of Palermo, Italy

Didem Gokcay,

Middle East Technical University,

Turkey

${ }^{*}$ Correspondence:

Yusuf O. Cakmak

yusuf.cakmak@otago.ac.nz

Received: 27 October 2016 Accepted: 12 June 2017

Published: 28 June 2017

Citation:

Cakmak YO, Apaydin H, Kiziltan G,

Gündüz $A$, Ozsoy $B$, Olcer $S$, Urey H, Cakmak OO, Ozdemir YG and Ertan S (2017) Rapid Alleviation of Parkinson's Disease Symptoms via Electrostimulation of Intrinsic Auricular

Muscle Zones.

Front. Hum. Neurosci. 11:338. doi: 10.3389/fnhum.2017.00338

\section{Rapid Alleviation of Parkinson's Disease Symptoms via Electrostimulation of Intrinsic Auricular Muscle Zones}

Yusuf O. Cakmak' ${ }^{*}$, Hülya Apaydin², Güneş Kiziltan², Ayşegül Gündüz², Burak Ozsoy³, Selim Olcer ${ }^{4}$, Hakan Urey ${ }^{4}$ Ozgur O. Cakmak ${ }^{5}$, Yasemin G. Ozdemir ${ }^{5}$ and Sibel Ertan ${ }^{2}$

1 Department of Anatomy, School of Medical Sciences, Otago University, Dunedin, New Zealand, ${ }^{2}$ Department of Neurology, Cerrahpasa School of Medicine, Istanbul University, Istanbul, Turkey, ${ }^{3}$ Global Dynamic Systems (GDS) ARGE, Teknopark Istanbul, Istanbul, Turkey, ${ }^{4}$ Department of Electrical Engineering, College of Engineering, Koç University, Istanbul, Turkey,

${ }^{5}$ Department of Neurology, School of Medicine, Koç University, Istanbul, Turkey

Background: Deep brain stimulation of the subthalamic nucleus (STN-DBS) and the pedunculopontine nucleus (PPN) significantly improve cardinal motor symptoms and postural instability and gait difficulty, respectively, in Parkinson's disease (PD).

Objective and Hypothesis: Intrinsic auricular muscle zones (IAMZs) allow the potential to simultaneously stimulate the C2 spinal nerve, the trigeminal nerve, the facial nerve, and sympathetic and parasympathetic nerves in addition to providing muscle feedback and control areas including the STN, the PPN and mesencephalic locomotor regions. Our aim was to observe the clinical responses to IAMZ stimulation in PD patients.

Method: Unilateral stimulation of an IAMZ, which includes muscle fibers for proprioception, the facial nerve, and C2, trigeminal and autonomic nerve fibers, at $130 \mathrm{~Hz}$ was performed in a placebo- and sham-controlled, double-blinded, within design, two-armed study of 24 PD patients.

Results: The results of the first arm (10 patients) of the present study demonstrated a substantial improvement in Unified Parkinson's Disease Ratings Scale (UPDRS) motor scores due to $10 \mathrm{~min}$ of IAMZ electrostimulation $(p=0.0003$, power: 0.99) compared to the placebo control $(p=0.130)$. A moderate to large clinical difference in the improvement in UPDRS motor scores was observed in the IAMZ electrostimulation group. The results of the second arm (14 patients) demonstrated significant improvements with dry needling $(p=0.011)$ and electrostimulation of the IAMZ $(\rho<0.001)$ but not with sham electrostimulation $(p=0.748)$. In addition, there was a significantly greater improvement in UPDRS motor scores in the IAMZ electrostimulation group compared to the IAMZ dry needling group $(p<0.001)$ and the sham electrostimulation $(p<0.001)$ groups. The improvement in UPDRS motor scores of the IAMZ electrostimulation group ( $\triangle \mathrm{UPDRS}=5.29$ ) reached moderate to high clinical significance, which was not the case for the dry needling group ( $\triangle$ UPDRS $=1.54$ ). In addition, both arms of the study demonstrated bilateral improvements in motor symptoms in response to unilateral IAMZ electrostimulation. 
Conclusion: The present study is the first demonstration of a potential role of IAMZ electrical stimulation in improving the clinical motor symptoms of PD patients in the short term.

Keywords: Parkinson's disease, electrostimulation, auricular muscles, PPN, STN, mesencephalon, locomotor, neuromodulation

\section{INTRODUCTION}

Bilateral high-frequency stimulation of the subthalamic nucleus (STN), commonly known as deep brain stimulation of the STN (STN-DBS), provides a significant improvement in cardinal motor symptoms and in the control of drug-induced complications of Parkinson's disease (PD). To be clinically effective, stimulation of the STN must be applied at frequencies greater than $100 \mathrm{~Hz}$ (Limousin et al., 1995; Limousin et al., 1997), and electrostimulation is usually delivered at $130 \mathrm{~Hz}$ (Little and Brown, 2012). Effective stimulation was shown to be associated with a significant decrease in the activity of the ipsilateral primary sensorimotor cortex at rest and a significant increase in premotor, anterior cingulate, pre-supplementary motor areas (pre-SMAs) and dorsolateral prefrontal cortices during movement (Payoux et al., 2004). On the other hand, axial symptoms, such postural instability and gait difficulty (PIGD), freezing of gait, and impaired speech, have been reported to be resistant to STN-DBS and levodopa treatments (Hamani et al., 2007; Tattersall et al., 2014). Recent clinical results have indicated the potential effectiveness of deep brain stimulation of the pedunculopontine nucleus (PPN-DBS) on PGID (Hamani et al., 2007; Tattersall et al., 2014). It has been demonstrated that lesions of the PPN induce gait deficits (Karachi et al., 2010; Tattersall et al., 2014). Moreover, cholinergic cell loss in the caudal PPN is accompanied by PIGD (Hirsch et al., 1987; Zweig et al., 1989; Karachi et al., 2010; Tattersall et al., 2014). Therefore, PIGD is thought to be related to the mesencephalic locomotor region rather than dopaminergic motor centers such as the STN (Garcia-Rill, 1991). The major components of the mesencephalic locomotor region are the PPN and the cuneiform nucleus (Garcia-Rill, 1991). In the context of different outcomes of STN-DBS and PPN-DBS, PPN dysfunction likely contributes to PIGD in PD (Tykocki et al., 2011). The PPN receives direct inputs from the pre-SMA and the basal ganglion to modulate muscle tone by directly exciting pontine reticular formation (Burn, 2013). Central pattern generators in the spinal cord can also be modulated directly and indirectly by the PPN (Figure 1B). Notably, sensory proprioceptive afferents have also been demonstrated to modify these patterns (Jankowska et al., 1983; Takakusaki et al., 2008; Burn, 2013).

Although altered proprioception is not a prominent symptom of PD, proprioception deficits have been reported in PD patients (Lee et al., 2013), and improvements in proprioception have been reported with STN stimulation (Lee et al., 2013). Selective muscle afferent nerve stimulation has been reported to cause significant activation in motor-related areas compared with cutaneous stimuli (Wardman et al., 2014). Muscle afferent stimulation evokes more widespread cortical, subcortical, and cerebellar activations than cutaneous afferents (Wardman et al., 2014). Separate precentral and post-central excitation foci were observed with muscle afferent stimulation, emphasizing the importance of muscle afferent nerve stimulation in the modulation of cortical motor areas (Wardman et al., 2014). Therefore, peripheral nerve stimulation may also hold the potential as an alternative and minimally invasive approach to modulate the activity in the premotor, anterior cingulate, pre-SMA and dorsolateral prefrontal cortices and basal ganglia such as the STN and the PPN and should be investigated in PD. Median nerve stimulation has been shown to result in the activation of STN neurons, which form a motor homunculus (Nambu et al., 1996, 1997, 2000; Hanajima et al., 2004). In theory, stimulation of other peripheral motor nerves may also activate motor homunculus of the STN.

Upper facial muscles around the orbital region are controlled bilaterally by the motor cortex through the facial nerve (Mima et al., 1999). In addition, unilateral STN-DBS has been demonstrated to induce strictly contralateral motor-evoked potentials in the trapezius, deltoid, biceps, and thenar muscles; however, the same stimulus reportedly always induces bilateral motor-evoked potentials in the orbicularis oculi, orbicularis oris, masseter, and sternocleidomastoid in Parkinson's patients (Costa et al., 2007). The intrinsic auricular muscles of the tragicus and antitragicus muscles have also been shown to simultaneously contract with the orbicularis oculi muscles (Matsuo and Hirose, 1987). The latter demonstration is also indirect proof of bilateral cortical connections of intrinsic auricular muscles, such as in the orbicularis oculi.

In the context of these anatomical relationships, unilateral stimulation of the intrinsic auricular muscle zones (IAMZs) may hold potential for bilateral feedback stimulation of muscle feedback and motor driver cortical areas. In addition to the possible proprioceptive outcomes of IAMZ stimulation, contributions of the facial nerve branches stimulating the intrinsic auricular muscles (Fujita, 1934; Matsuo and Hirose, 1987), the vagus nerve via the Arnold branch as a parasympathetic contribution (Alvord and Farmer, 1997; Peuker and Filler, 2002), the trigeminal nerve via the auriculotemporal nerve branch (Alvord and Farmer, 1997; Peuker and Filler, 2002) and the C2 spinal nerve within the great auricular nerve (Alvord and Farmer, 1997; Peuker and Filler, 2002) to IAMZ have been demonstrated in the literature (Figure 1A). In addition, cervical sympathetic nerves are distributed to the auricula within the ear arteries (Daniel and Paton, 1975; Lambru et al., 2013) (Figure 1A). Therefore, stimulation of the IAMZ has the potential for synchronous stimulation of the $\mathrm{C} 2$ spinal nerve, the trigeminal nerve, and sympathetic and parasympathetic nerves (Figure 1A), each of which may contribute to motor regulation. 

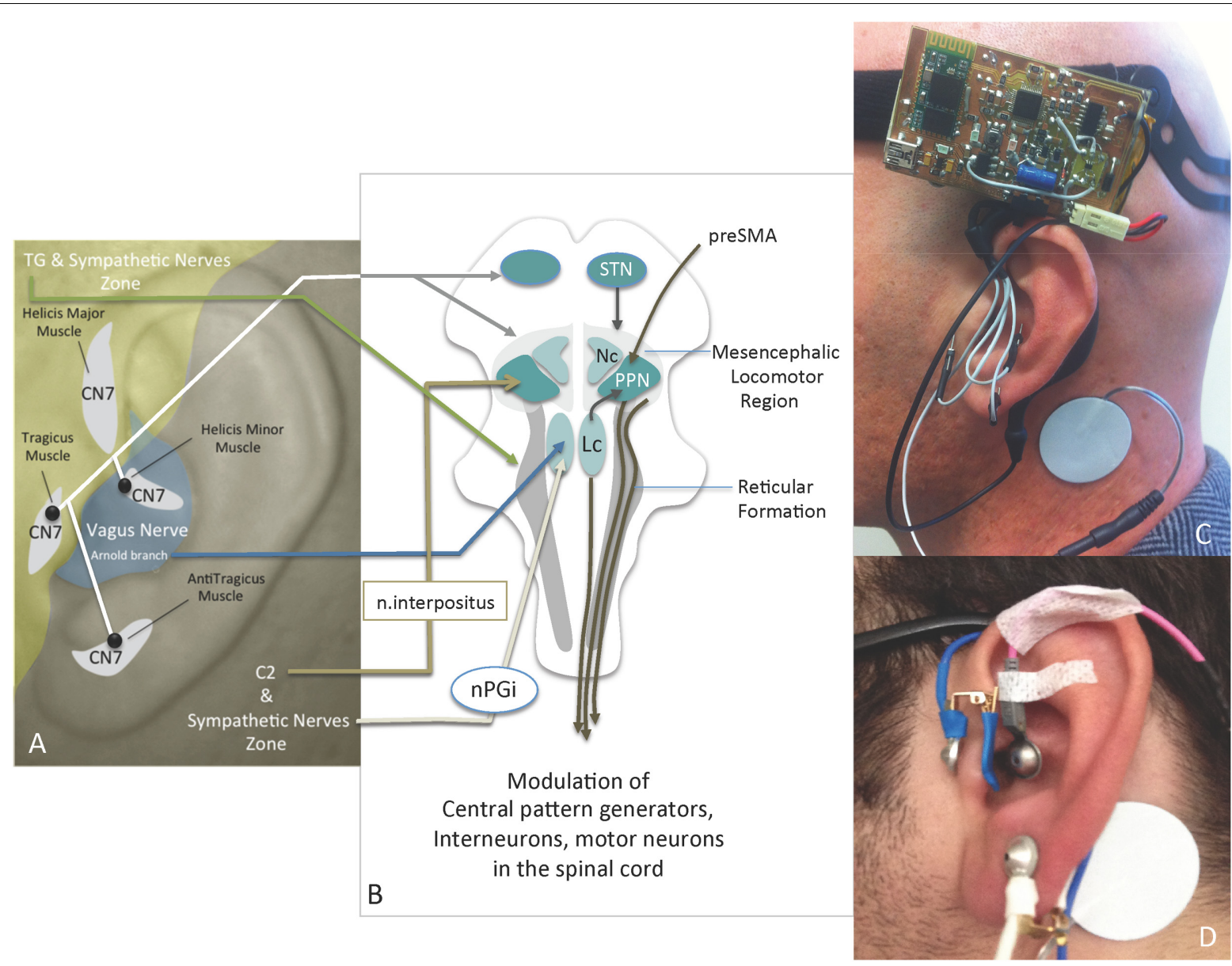

FIGURE 1 | (A) Innervation of the ear, the IAMZs and IAMZ electrode locations (black dots); (B) relevant anatomical pathways of IAMZs via auricular nerves for motion regulation and mesencephalic locomotor regions (modified from Burn, 2013); (C) active stimulation with needle electrodes of the IAMZ and with TENS electrode of the SCM; (D) placebo group TENS electrode placements in the IAMZ and SCM; CN7, facial nerve; C2, C2 spinal nerve; TG, trigeminal nerve; Nc, nucleus cuneatus; Lc, locus coerulei; nPGi, nucleus paragigantocellularis; STN, subthalamic nucleus; PPN, pedunculopontine nucleus. Yellow zone: trigeminal nerve + sympathetic nerve zone; Gray zone: C2 + sympathetic nerve zone; White areas: intrinsic auricular muscles zones. Blue area: Arnold branch of the vagus nerve, $\mathrm{C} 2, \mathrm{CN7}$, and sympathetic nerves overlapping zone.

It has been demonstrated that the $\mathrm{C} 2$ spinal nerve forms anatomical connections with the deep cerebellar nucleus of the interpositus (represented by the nucleus globosus and the emboliformis in humans) in animals (Matsushita and Xiong, 2001). The interpositus has projections to the PPN and from the PPN to the STN (Schell and Strick, 1984; Hazrati and Parent, 1992; Lavoie and Parent, 1994; Harting, 1997; McFarland and Haber, 2000; Muthusamy et al., 2007). In addition, the C2interpositus nucleus-PPN-reticulospinal axis may play a cardinal role in motion and position regulation: a substantial body of evidence suggests that the reticulospinal system is essential and plays a fundamental role in the integration of commands for whole-body movement and postural adjustment rather than the movement of a single limb (Magni and Willis, 1964; Keizer and Kuypers, 1984, 1989; Drew and Rossignol, 1990; Luccarini et al., 1990; Drew, 1991; Massion, 1992; Mori et al., 1992; Kably and Drew, 1998; Schepens and Drew, 2003) (Figure 1B). Of note, the reticulospinal system is also directly excited by the PPN and mediates DBS-PPN to modulate muscle tone in PD patients (Burn, 2013) (Figure 1B). In addition to the potential effects of the $\mathrm{C} 2$ spinal nerve on the reticulospinal system via the interpositus nucleus-PPN axis, the mesencephalic trigeminal nucleus as the principal nucleus of the facial muscles' proprioceptive center, projects to the reticular formation and to the spinal cord via propriospinal neurons (Lawrence and Kuypers, 1968; Gorska and Sybirska, 1980; Alstermark et al., 1981, 1989; Hobbs et al., 1992; Usunoff et al., 1997; Whishaw et al., 1998; Sasaki, 2004; Jankowska and Edgley, 2006) (Figures 1A,B). This connection also underlines the potential role of intrinsic auricular muscle proprioception feedback on reticular formation and the possible influences on movement and posture, in addition to C2-related pathways, on reticular formation and propriospinal neurons. The $\mathrm{C} 2$ spinal nerve projects to the lower ear lobe and the corresponding skin that overlays the intrinsic muscles of the antitragicus muscle by the greater auricular nerve and the trigeminal nerve; its auriculotemporal branch innervates the skin 
areas over the tragicus and helicis major muscles (Matsuo and Hirose, 1987; Alvord and Farmer, 1997; Peuker and Filler, 2002; Lambru et al., 2013; Drake et al., 2015) (Figure 1A).

In addition to the possible indirect autonomic effects of IAMZ stimulation via the $\mathrm{C} 2$ nerve, autonomic fibers of the auricula also directly contribute to these zones. While the sympathetic nerves overlay the IAMZ within the arterial walls, the Arnold branch of the vagus nerve is distributed only over the helicis minor muscle zone (Matsuo and Hirose, 1987; Alvord and Farmer, 1997; Peuker and Filler, 2002; Lambru et al., 2013; Drake et al., 2015) (Figure 1A). Thus far, no neuromodulation studies of the Arnold branch or neurotraces of the efferent connections of the Arnold branch have considered the existence of the helicis minor muscle in the concha area where the Arnold branch is distributed.

The earliest accumulation of $\alpha$-synuclein and Lewy bodies have been shown to occur in the dorsal motor nucleus of the vagus nerve in PD (Braak et al., 2003). A recent study also showed that chronic impairment of vagus nerve function leads to inhibition of dopamine and that low frequency stimulation of the vagus nerve significantly inhibited the dopamine system in rat brain structures (Ziomber et al., 2012). It is worth noting that low- and high-frequency electrostimulation induce opposite outcomes on the autonomic nerve system and neurotransmitters (Cakmak et al., 2008, 2016; Zhao, 2008), so that high frequency of stimulation may result with the stimulation of the dopaminergic system.

In the context of the demonstrated structural and functional anatomical connections of the IAMZ and the underlined mechanisms of deep brain stimulation studies in PD, the IAMZ is the only potential auricular zone to synchronously stimulate the C2 spinal nerve, the trigeminal nerve, autonomic nerve fibers and proprioceptive centers to modulate motor modulatory centers including mesencephalic locomotor region. We hypothesized that the stimulation of IAMZ would be beneficial to alleviate the PD motor symptoms. A clinical trial is designed to investigate the potential clinical outcomes (motor symptoms) of the IAMZ stimulation in PD patients. We stimulated the IAMZ (which includes the helicis minor, tragicus, and antitragicus muscles) unilaterally (ipsilateral to the dominant PD symptoms) at a high frequency and analyzed the efficacy of this technique on 24 PD patients via a double-blind, placebo- and sham controlled within-subject design, two-armed study.

\section{MATERIALS AND METHODS}

All subjects provided written informed consent to undergo the procedure. The study was approved by the Ethics Committee of the Koç University, Turkey, and it was carried out in accordance with the Ethical Principles for Medical Research Involving Human (Declaration of Helsinki).

Koç University Clinical Trials Ethics Committee Approval Number: 2015.091.IRB1.018, ClinicalTrials.gov Identifier: NCT02722824,

Turkey Ministry of Health: Follow up number - 133235.

\section{Research Participants}

Ten volunteer patients with idiopathic PD who had been followed in our movement disorders outpatient clinic were enrolled for the first arm of the study. Another 14 volunteer patients with idiopathic PD with the same criteria were enrolled for the second arm of the study. PD diagnoses were made by a neurologist who was an expert in movement disorders using the UK Parkinson's Disease Society Brain Bank clinical diagnostic criteria (Hughes et al., 1992).

All patients underwent a detailed neurological examination, and parkinsonian features were rated according to the Unified Parkinson's Disease Rating Scale (UPDRS) Part III. Patients with a disease duration longer than 2 years and Hoehn and Yahr stage $\geq 2$ were included. Patients with cognitive impairment that might prevent cooperation during tests and patients with any other neurological or systemic disease in which electrostimulation was contraindicated were excluded.

\section{Electrostimulation and Analysis of Symptoms}

Arm 1 [10 patients - 10 min of electrostimulation of the IAMZ with needle electrodes and 10 min of placebo using transcutaneous electrodes (TENS) for IAMZ]: two movement disorder specialists blind to the nature of stimulation rated all patients twice according to the motor section of the UPDRS just before the stimulation (baseline evaluation) and $10 \mathrm{~min}$ after the onset of stimulation or placebo application. The patients and two movement disorder specialists were informed that there would be two modes of stimulation, sub-threshold stimulation with TENS of the IAMZ (placebo group, Figure 1D) and above the sensory threshold with needle electrodes of the IAMZ (active group, Figure 1C). In the placebo-control application in which there was no stimulation and the device was in off-mode, the patients and two movement disorder specialists were informed that a new device would be placed on the ear to improve their motor symptoms and that they would not sense the stimulation of the device because of the sub-threshold stimulation of the IAMZ via the TENS. In the active group, the patients and two movement disorder specialists were informed that the stimulation would be above the sensory threshold and applied via the needle electrodes to the IAMZ. In both groups, there was a TENS electrode on the sternocleidomastoid muscle (SCM) (Figures 1C,D). The consort flow chart (Figure 2) demonstrates the study design in the first arm.

Arm 2 [14 patients - 20 min of stimulation in three sessions: electrostimulation of the IAMZ with needles, needling of the IAMZ without electrostimulation (dry needling) and sham region electrostimulation with needles]: the main aims of the second arm of the study were to assess the following:

- a potential effect of dry needling on the IAMZ without electrostimulation (dry needling of the IAMZ zones without electrostimulation but keeping the needle electrodes connected with to the electrostimulator),

- the potential effect of stimulation of only the C2/sympathetic nerve by ensuring no or minimal 


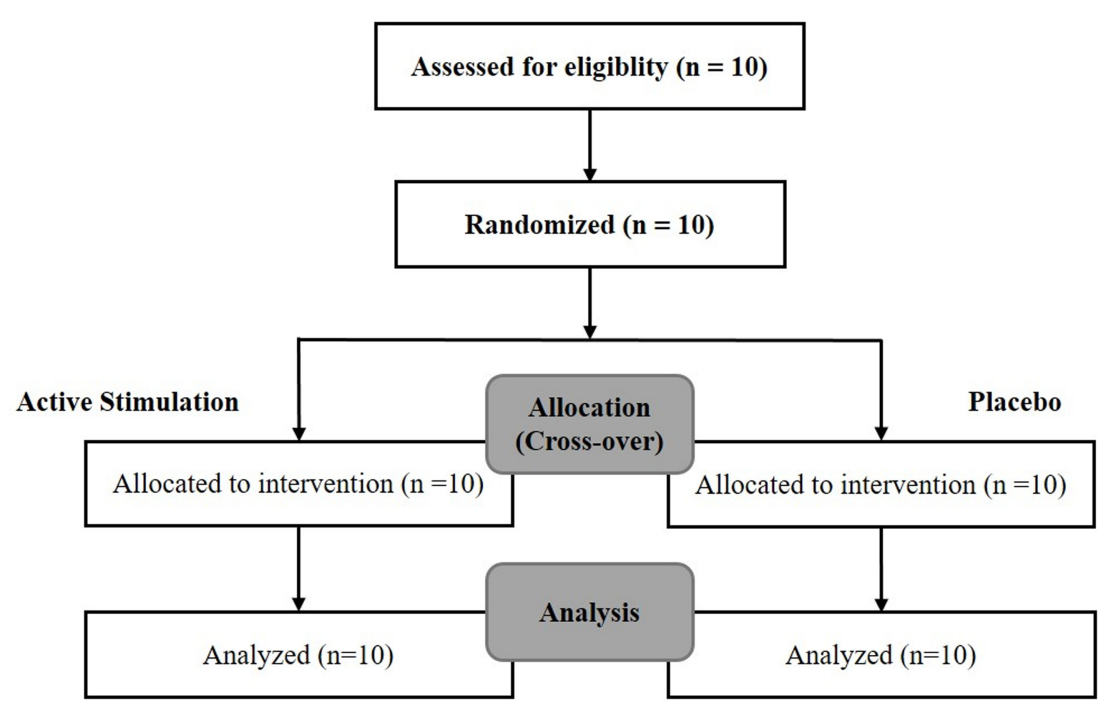

FIGURE 2 | The consort flow chart of the first arm of the study.

stimulation of the SCM, the vagus facial nerve and the trigeminal nerve (sham stimulation),

- the specificity of the IAMZ zone electrostimulation (active group-IAMZ electrostimulation),

- the sole specificity of $130 \mathrm{~Hz}$ stimulation (sham stimulation zone),

- a potential long-term effect of the stimulation (50thminute).

In the second arm of the study, the stimulation duration proceeded to the 20th-minute, and patients were assessed at the 50th-minute (30 min after the termination of 20min stimulation). The two movement disorder specialists who participated in the first arm and who were blind to the nature of stimulation rated all $(n=24)$ patients twice according to the motor section of the UPDRS.

The two movement disorder specialists were blinded to the type of stimulation by covering the entire auricula with cotton pads, including the needle electrodes (Figure 3A). Patients were also informed that there would be three modes of stimulation: sub-threshold stimulation with needles (dry needling of the IAMZ without electrostimulation but with the needles connected to stimulator as in the needle electrostimulation group, Figure 1C) and electrostimulation with needle electrodes above the sensory threshold at two different auricular zones (electrostimulation with needle electrodes on the IAMZ Figure $\mathbf{1 C}$ and a sham region Figure $3 \mathbf{B}$ ). These groups ensured that the patients were blind to the active stimulation group. A part of the upper helix that is innervated by $\mathrm{C} 2 /$ sympathetic nerves but free or with minimal contributions of the SCM, vagal, facial, and trigeminal nerves was selected as the sham electrostimulation region (Figure 3B). The consort flow chart in Figure $\mathbf{4}$ demonstrates the study flow in the second arm.

\section{Stimulation Procedure}

All examinations and electrostimulations were performed during "off" periods of the patients (when the effect of the dopaminergic drug was minimal). We designed and built an electrical signal generator with a changeable voltage output (0-5 V), frequency $(2-300 \mathrm{~Hz})$ and pulse length $(50-200 \mu \mathrm{s})$ with external electrodes able to be connected to press needles (KINGLI, China) placed on the three intrinsic auricular muscles (tragicus, antitragicus, and helicis minor muscles). While the needle electrodes were placed on the IAMZ (Figure 1C) in the active stimulation and dry needling groups and over the upper helix in the sham stimulation group (Figure 3B), the transcutaneous electrode was placed on the SCM $1-2 \mathrm{~cm}$ to its mastoid origin (Figure 1C) near the electrical stimulation circuit. In the sham group, the transcutaneous electrode was placed but remained inactive to keep the electrical field of stimulation in the selected helix region for $\mathrm{C} 2 /$ sympathetic nerve stimulation. It was also placed and kept inactive in the dry needling group to maintain observer blindness.

The SCM was chosen for surface electrode placement for three reasons:

(1) STN contribution: bilateral SCM activation has been reported during unilateral STN stimulation, which underlines the bilateral STN connections to each SCM (Costa et al., 2007).

(2) Nucleus cuneatus contribution: the mesencephalic locomotor region has two major components: the PPN and the nucleus cuneatus. SCM proprioception projects to the nucleus cuneatus as the neck muscle proprioception center [6] and as a mesencephalic locomotor component (Garcia-Rill, 1991) (Figure 1B).

(3) C2 spinal nerve contribution: the skin over the SCM $(1-2 \mathrm{~cm}$ to its mastoid origin) is also innervated by the $\mathrm{C} 2$ spinal nerve (Matsuo and Hirose, 1987; Alvord and Farmer, 1997; 


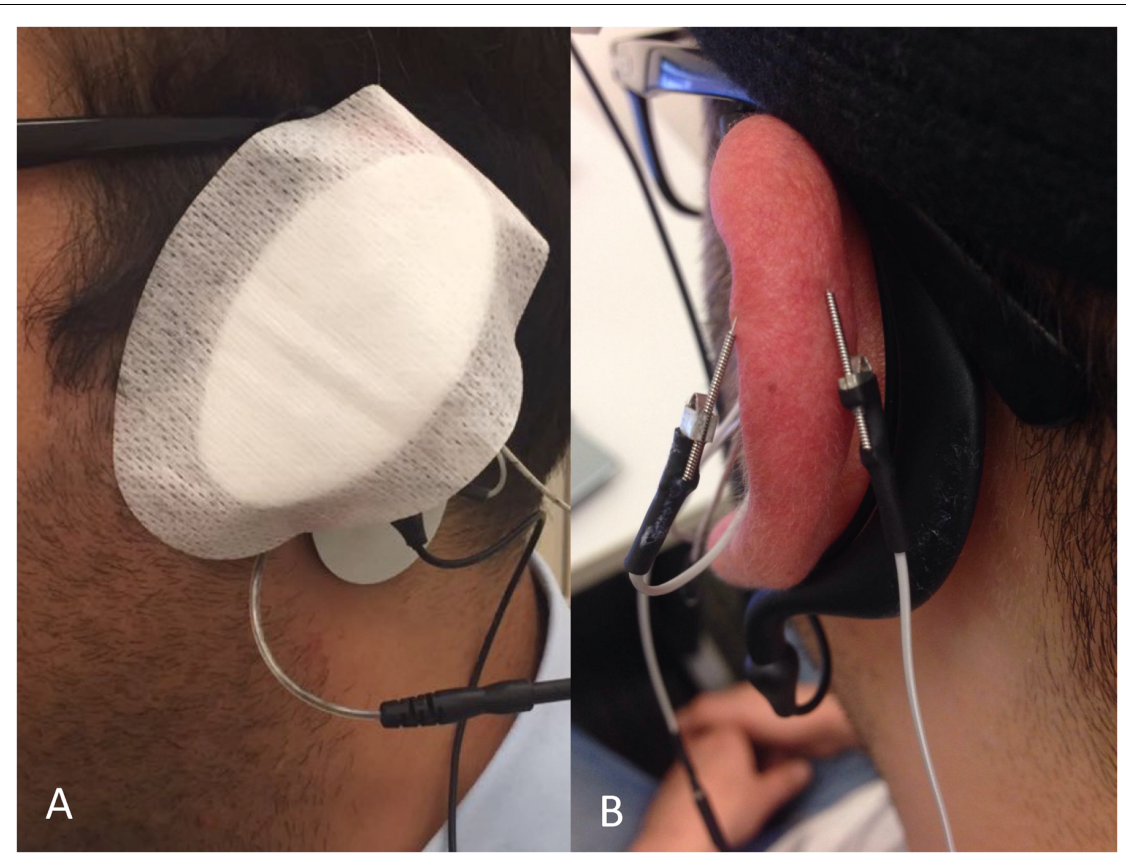

FIGURE 3 | (A) A pad covered the ear to ensure observer blindness in the groups of the second arm of the study. (B) Sham stimulation zone needle electrode placement.

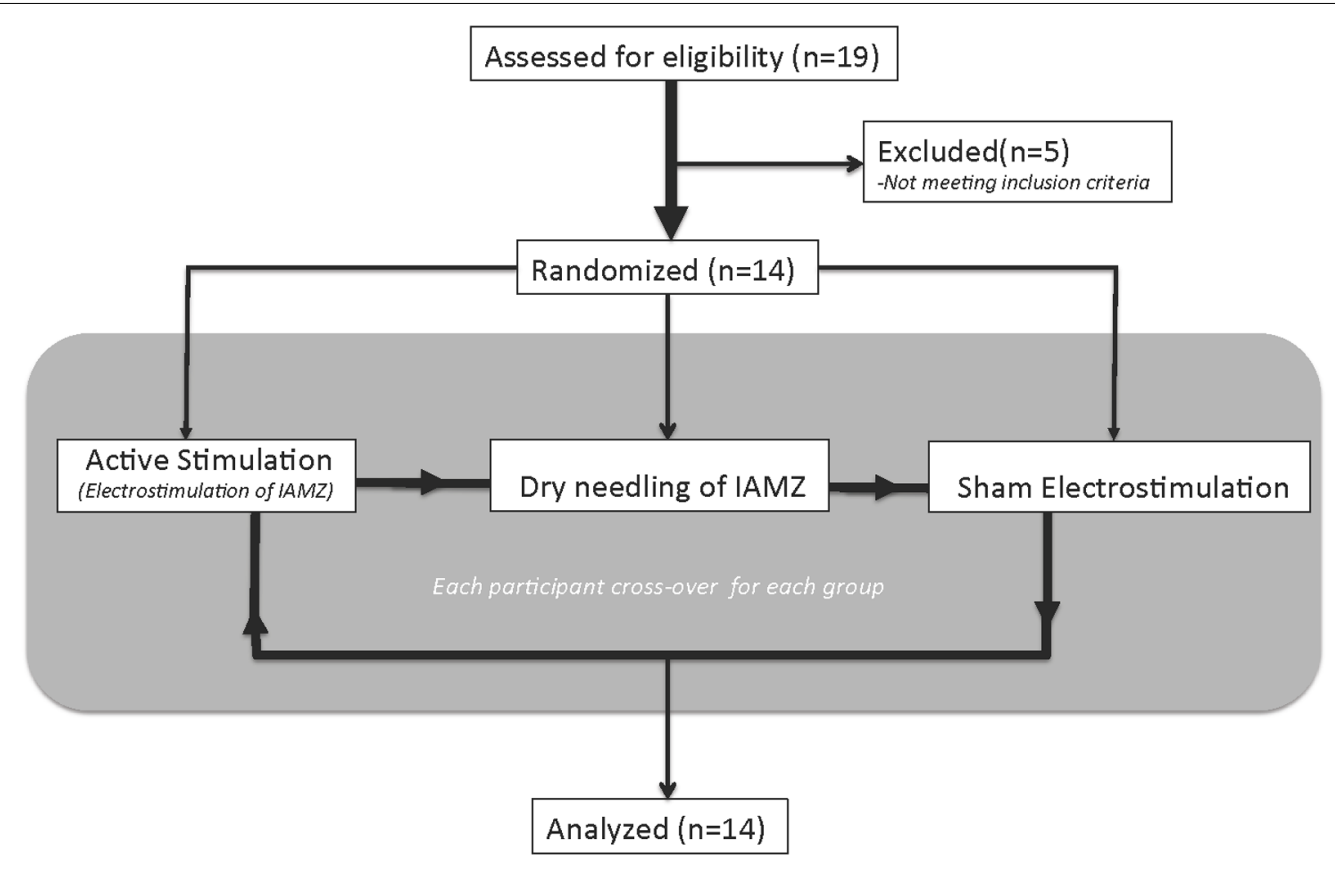

FIGURE 4 | The consort flow chart of the second arm of the study.

Peuker and Filler, 2002; Lambru et al., 2013; Drake et al., 2015) (Figure 1A).

In the present study, we used a biphasic wave-form and a stimulation style that alternated the anode and cathode for each stimulus during the stimulation period such that the needle electrodes on the intrinsic auricular muscles and the surface electrode on the SCM served as both the anode and cathode in an alternating manner. Given the much larger contact surface of the transcutaneous electrode compared with the needle electrodes and taking into account the difference in the skin conductance between the needle electrode and the transcutaneous electrode 
in addition to the low power of the stimulus, the transcutaneous electrode on the SCM would have a minimal impact on the stimulation-related outcomes.

Auricular electrical stimulation was applied with the following STN-DBS parameters: $130 \mathrm{~Hz}$ with a wavelength of $100 \mu \mathrm{s}$ and an intensity just under the pain threshold (1-4 V). The stimulation was administered unilaterally (ipsilateral to the side of prominent symptoms). We did not set a power (V) for the stimulation because such an approach does not eliminate the probability of current differences due to skin conductance differences in different subjects or within subjects during different sessions. To overcome this possible problem, the electrostimulator contained a built-in current-control feature so that the current was stabilized in the range of 100-130 $\mu \AA$ during the stimulation period by modulating the power and ensuring that the tingling sensation was under the pain threshold. This approach overcomes the interindividual and intraindividual (in active and control sessions) differences in skin conductance.

In addition to the traditional approach of taking into account the dominant symptom side scores of lateralized subitems (items 20-26) for the UPDRS Part-III motor scale scoring, the scores of lateralized subitems on both sides (ipsilateral and contralateral to the dominant symptoms and to the stimulator) were also documented to reveal any potential effects of unilateral stimulation over the bilateral motor symptoms. Moreover, subscores of the UPDRS Part-III were also classified and analyzed as Tremor (items 20-21), Rigidity (item 22), Bradykinesia (items 23-26,31), Gait and Postural Stability (items 27-30) and Bulbar Anomalies (items 18-19), as in previous studies (Postuma et al., 2008). All statistical analyses were performed using Student's paired $t$-tests (Prism 7 demo version, GraphPad Software, Inc., La Jolla, CA, United States, 2016). In addition, the Bonferroni correction was also applied as a conservative approach for the subscore group analysis $[n=2$ (active and placebo), $p<0.025$ in Arm 1, and $n=3$ (active, dry needling, and sham), $p<0.016$ in Arm 2]. The second arm of the study, which included three groups, also analyzed via analysis of variance (ANOVA) for group comparisons.

\section{RESULTS}

\section{Arm 1}

The mean age of the patients was $55.7 \pm \mathrm{SD} 7.8$ years, and only one patient was female. The mean disease duration was $8.3 \pm$ SD 4.4 years. Detailed clinical features of the patients are presented in Table 1.

There were no statistically significant differences between the baseline UPDRS motor scores of the patients in the active and placebo groups $(p=0.735)$. The UPDRS motor scores showed a statistically significant improvement $10 \mathrm{~min}$ after auricular stimulation compared with baseline UPDRS scores $(p=0.0003$, power: 0.99$)$. The placebo group did not show statistically significant differences in UPDRS motor scores compared with baseline $(p=0.130)$. At the 10th-minute, the mean improvement rates were $35 \%(\triangle \mathrm{UPDRS}=5.9)$ in the active group [mean UPDRS motor scores: 17.0 at baseline, 11.1

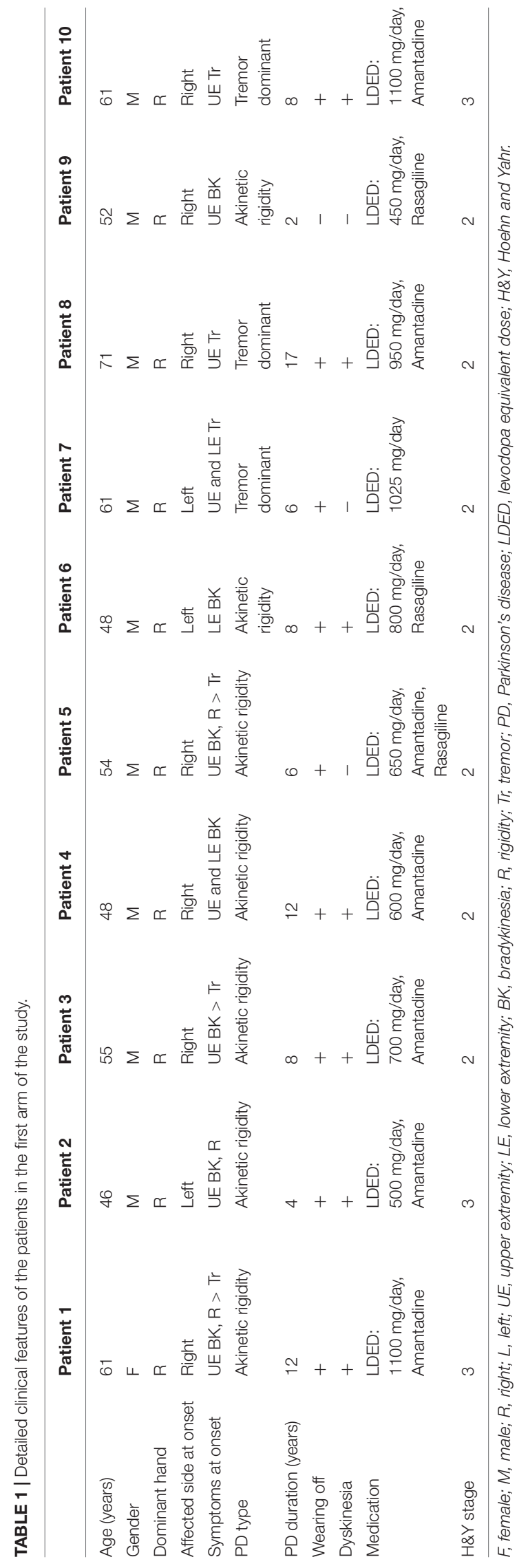


at the 10th-minute, standard deviation (SD): 3.6 at baseline and 4.6 at the 10 th-minute, $\triangle$ UPDRS range $=1.5-12]$ and $5 \%$ $(\triangle \mathrm{UPDRS}=1)$ in the placebo-control group (mean UPDRS motor scores: 17.4 at baseline, 16.4 at the 10th-minute, $S D$ : 4.6 at baseline and 4.5 at the 10th-minute, $\triangle \mathrm{UPDRS}$ range $=$ $-1.5-4.5)$.

Figure 5 shows the UPDRS Part-III scores and the group comparison for each case and an overall summary of the first arm. Figure 6 summarizes the classified UPDRS subscore differences for the group comparisons with $t$-tests, Figure 7 gives these differences for the group comparisons after the Bonferroni correction. Table 2 summarizes the $p$-values for the $t$-tests and after the Bonferroni correction of the classified UPDRS subscores for the 10th-minute compared with baseline.

The bilateral assessments of the symptomatic improvements of PD patients before and after electrical stimulation revealed that unilateral stimulation of the intrinsic auricular muscles can relieve $\mathrm{PD}$ symptoms on both sides at the 10th-minute. The 10th-minute mean improvements in the lateralized (UPDRS Part III subscore 20-26) UPDRS motor subscores were 3.0 on the contralateral side and 3.2 on the ipsilateral side in the active
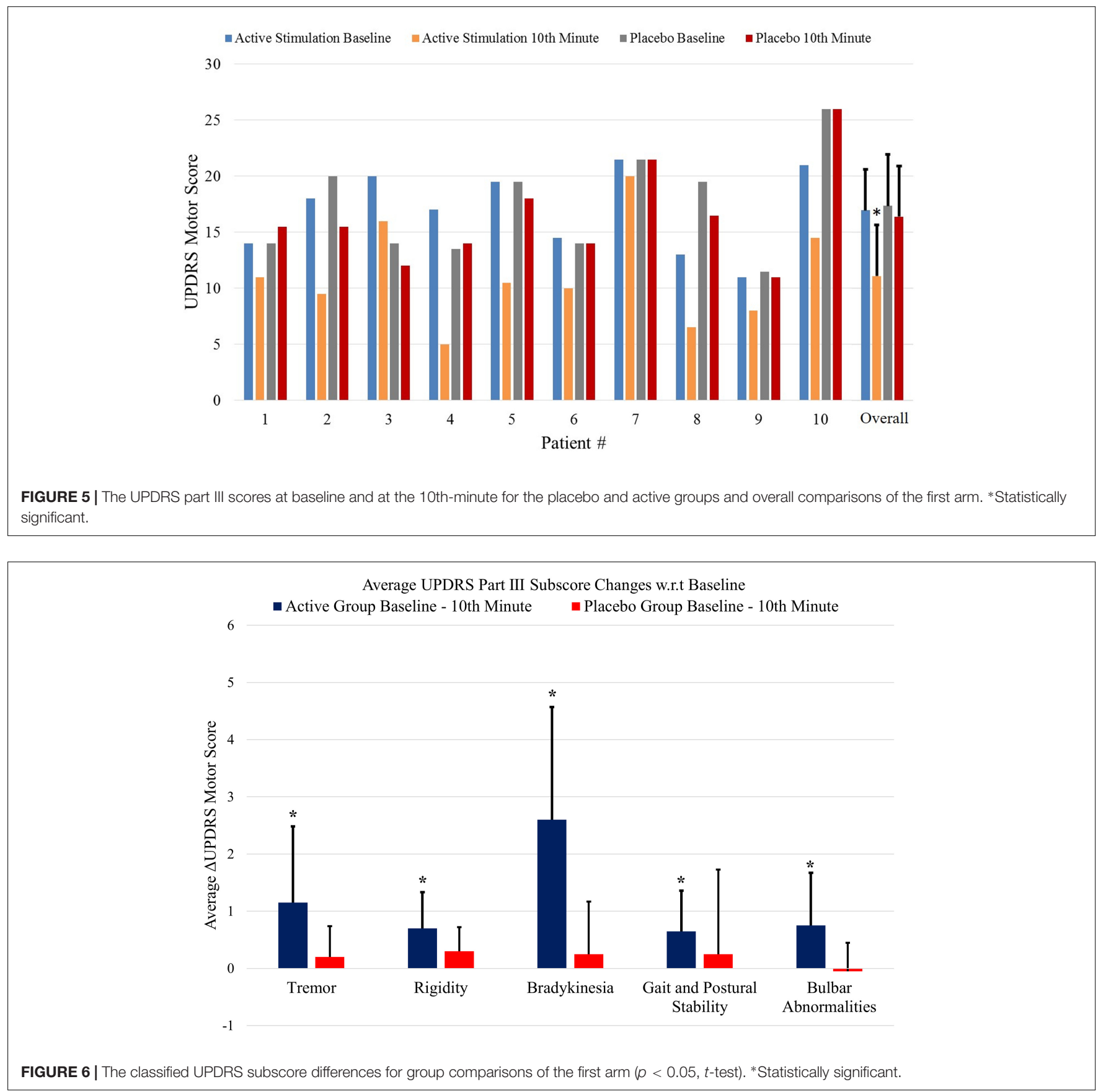


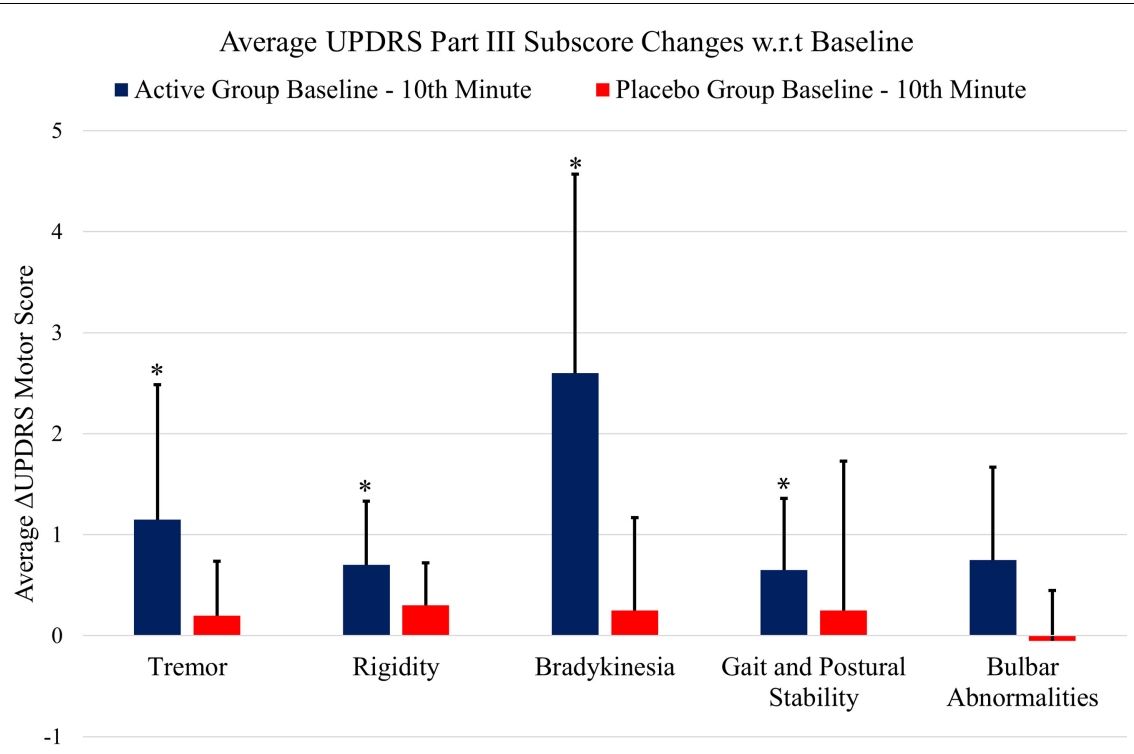

FIGURE 7 | The classified UPDRS subscore differences for group comparisons with Bonferroni correction. *Statistically significant.

TABLE 2 | The $p$-values after Bonferroni correction of the classified UPDRS subscores for the 10th-minute compared with baseline of the first arm study groups.

\begin{tabular}{lcc}
\hline Subgroup & $\boldsymbol{p}$-Value active group & $\boldsymbol{p}$-Value placebo group \\
\hline Tremor & $\mathbf{0 . 0 2 3}$ & 0.398 \\
Rigidity & $\mathbf{0 . 0 0 7}$ & 0.051 \\
Bradykinesia & $\mathbf{0 . 0 0 2}$ & 0.413 \\
Gait and postural instability & $\mathbf{0 . 0 1 8}$ & 0.605 \\
Bulbar abnormalities & $\mathbf{0 . 0 3 0}$ & 0.758
\end{tabular}

t-test; $p<0.05$, Bonferroni correction $p<0.025$, Italic: significant in t-test, bold: significant after Bonferroni correction, bold and italic: significant in t-test and Bonferroni.

group. The 10th-minute difference in the lateralized UPDRS motor subscores in the placebo-control group were 0.5 on the contralateral side and 0.8 on the ipsilateral side.

\section{Arm 2}

The mean age of the patients was $58.1 \pm$ SD 9.8 years, and 6 of the patients were female. The mean disease duration was $7.9 \pm$ SD 3.7 years. Detailed clinical features of the patients are presented in Table 3. Baseline UPDRS motor scores of the three groups were compared via one-way repeated measures ANOVA. There were no statistically significant differences between the baseline UPDRS motor scores of the patients in the active (IAMZ electrostimulation), IAMZ dry needling and sham electrostimulation groups prior to the interventions (pairwise comparison $p$-values: active vs. dry needling: 0.286 , active vs. sham: 0.052, sham vs. dry needling: 1.0).

In the active group, the UPDRS motor scores were significantly improved at the 50th-minute after the initiation of IAMZ electrostimulation (i.e., $30 \mathrm{~min}$ after the termination of the 20-min stimulation) compared with baseline UPDRS scores ( $p<0.001$, power: 0.99). The IAMZ dry needling group also showed statistically significant differences in UPDRS motor scores after the dry needling procedure compared with baseline $(p=0.011)$. However, the improvement in UPDRS motor scores of dry needling stimulation of the IAMZ ( $\triangle$ UPDRS $=1.54$ ) did not reach clinical significance (Shulman et al., 2010) as electrostimulation of the IAMZ ( $\triangle$ UPDRS $=5.29)$. The improvement in the UPDRS score observed in the active group (electrostimulation of the IAMZ) was in the range of moderate to high clinical significance (Shulman et al., 2010). There was no significant difference in UPDRS motor scores in the sham electrostimulation group $(p=0.748)$.

The mean improvement rates at the 50th-minute were $31 \%$ ( $\triangle$ UPDRS $=5.29$ ) in the active group (mean UPDRS motor scores: 17.71 at baseline, 12.42 at the 50th-minute, SD: 4.27 at baseline and 4.61 at the 50th-minute, $\triangle$ UPDRS range $=0.50-$ 9.50), $9 \%(\triangle \mathrm{UPDRS}=1.54)$ in the dry needling group (mean UPDRS motor scores: 16.04 at baseline, 14.50 at the 50thminute, $S D: 3.1$ at baseline and 3.3 at the 50th-minute, $\triangle$ UPDRS range $=0.50-7.0)$ and $0 \%(\triangle \mathrm{UPDRS}=0.20)$ in the sham stimulation group (mean UPDRS motor scores: 15.25 at baseline, 15.05 at the 50th-minute, SD: 5.09 at baseline and 5.15 at the 50th-minute, $\triangle \mathrm{UPDRS}$ range $=-3.5$ to 4.0 ).

One-way repeated measures ANOVA was also performed for the difference in each group's baseline and post-stimulation UPDRS scores ( $\triangle$ UPDRS comparison). The $\triangle$ UPDRS comparison for the active vs. dry needling groups and the active vs. sham groups were significant $(p<0.001$ for both comparisons); however, the comparison of $\triangle$ UPDRS for the sham vs. dry needling groups was not statistically significant $(p=0.319)$.

Figure 8 gives the UPDRS Part-III scores and the group comparison for each case and the overall summary of the second arm. Figure 9 summarizes the classified UPDRS subscore 


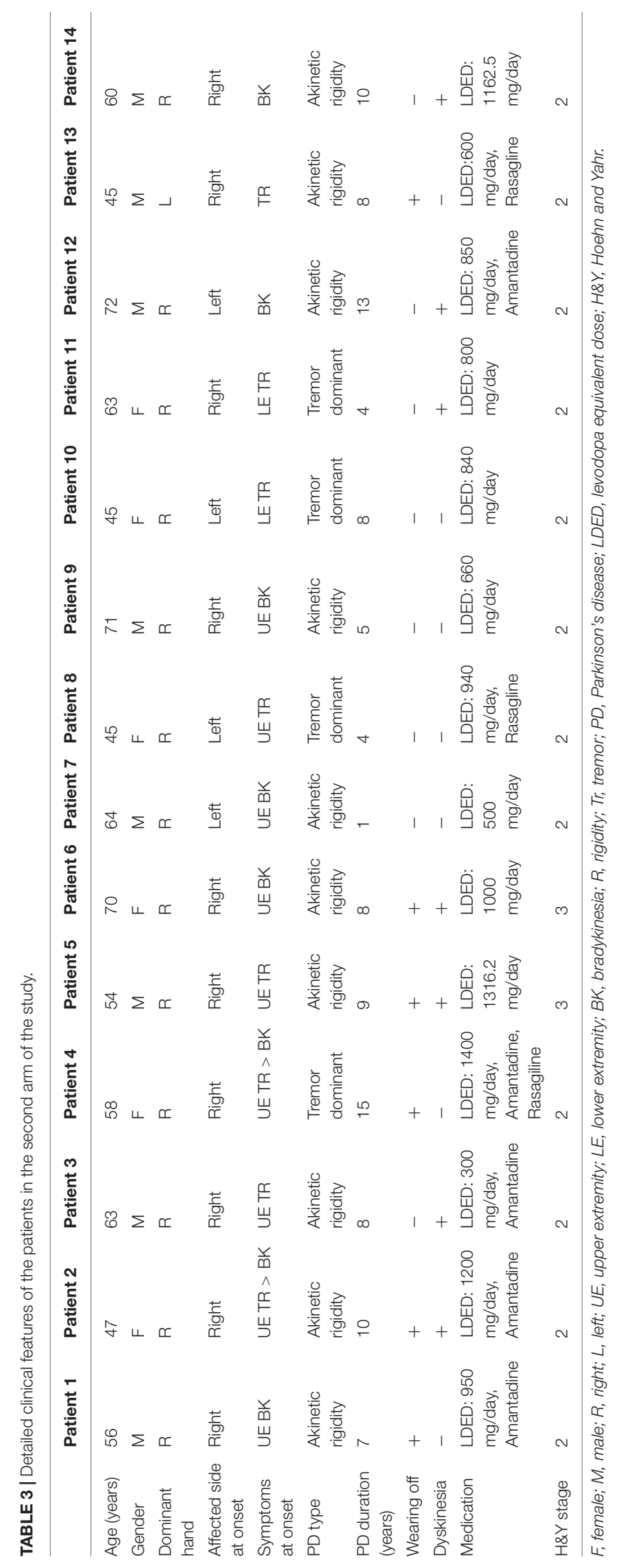




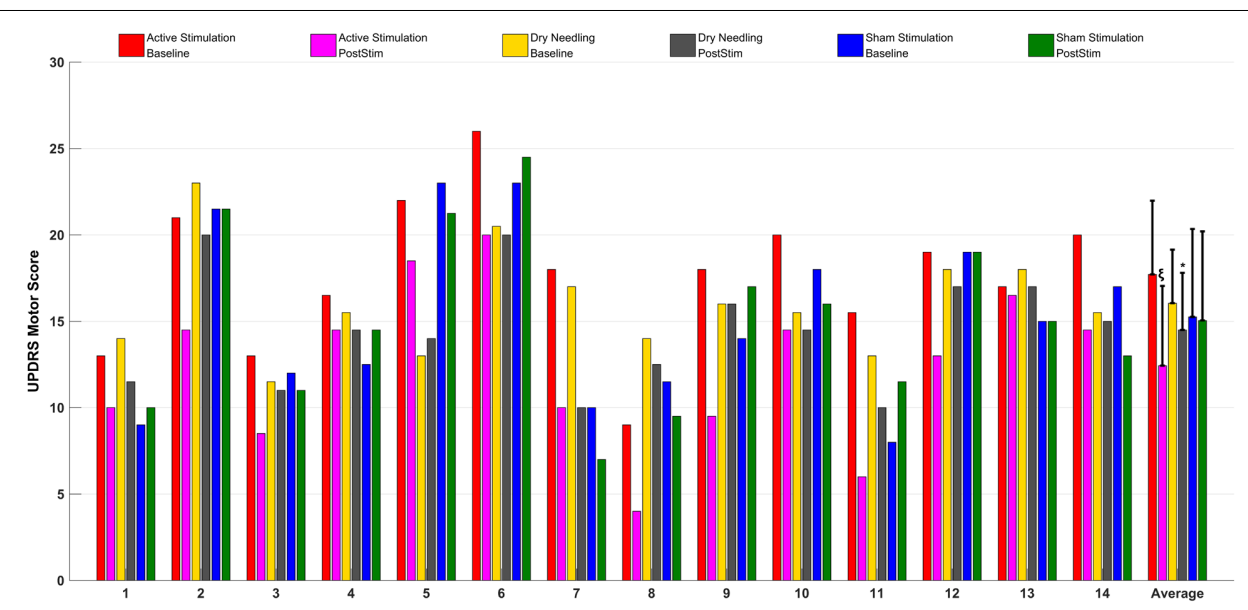

FIGURE 8 | The UPDRS part III scores at baseline and at the 50th-minute for the active group (electrostimulation of the IAMZ), IAMZ dry needling group, and sham stimulation group and overall comparisons of the second arm. *Statistically significant, $\xi$ statistically and clinically significant.

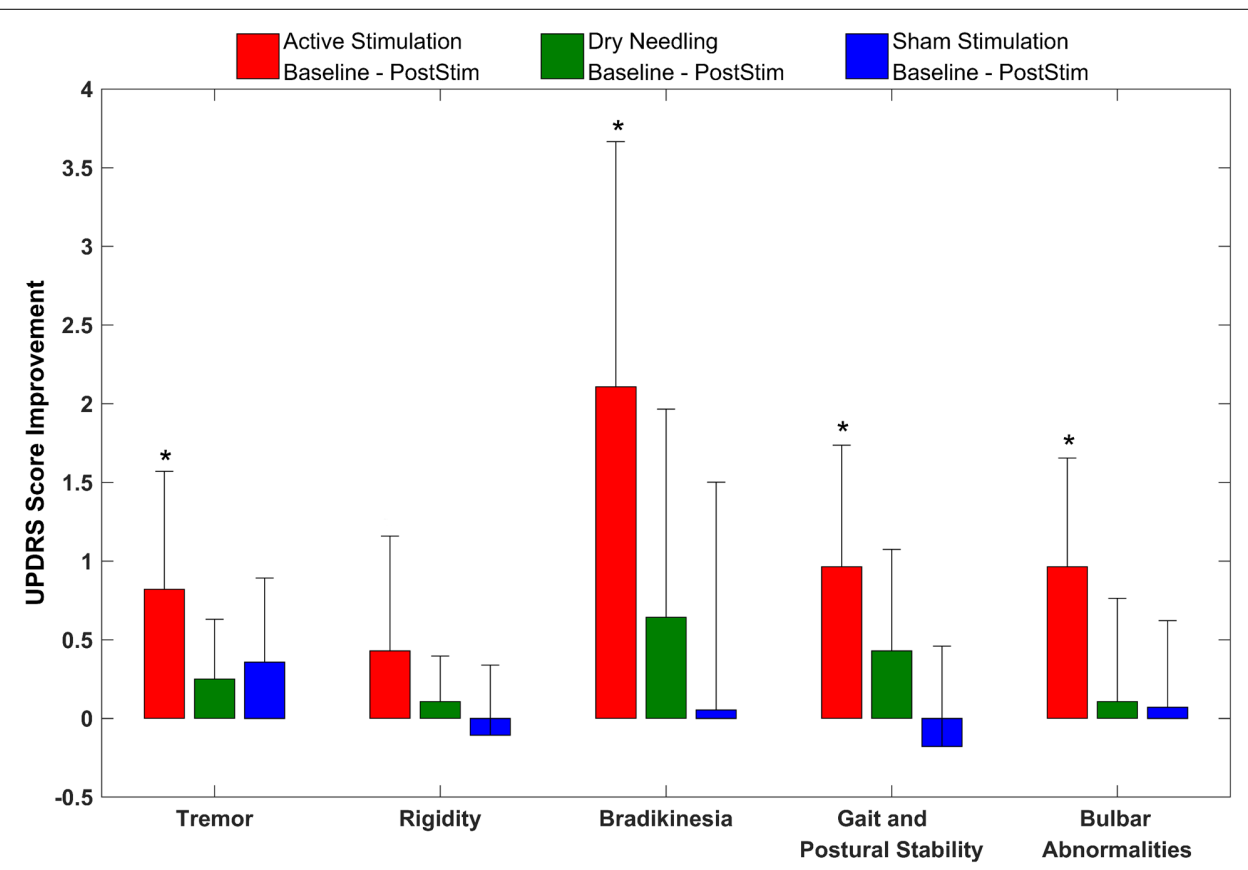

FIGURE 9 | The classified UPDRS subscore differences for group comparisons with Bonferroni correction of the second arm of the study. ${ }^{*}$ Statistically significant.

differences in the group comparisons with Bonferroni correction. Table 4 summarizes the $p$-values for $t$-tests and after Bonferroni correction of the classified UPDRS subscores for the 50th-minute compared with baseline.

Bilateral assessments of the symptomatic improvements of PD patients before and after electrical stimulation of the IAMZ revealed that unilateral stimulation of the intrinsic auricular muscles can relieve PD symptoms on both sides at the 50thminute. The 50th-minute mean improvements in the lateralized (UPDRS Part III subscore 20-26) UPDRS motor subscores were 2.39 on the contralateral side and 1.89 on the ipsilateral side in the active group. The 50th-minute differences in the contralateral and ipsilateral UPDRS motor subscores were 0.79 and 0.61 in the dry needling group and 0.48 and 0.0 in the sham group, respectively.

\section{DISCUSSION}

The results of the present study demonstrated a substantial improvement in UPDRS motor scores after electrostimulation of the IAMZ but not after placebo or sham stimulation. The UPDRS score improvements in the electrostimulation of IAMZ groups were statistically and clinically significant. Comparison of the $\triangle$ UPDRS (5.9 in the first arm and 5.29 in the second arm) of 
TABLE 4 | The $p$-values after Bonferroni correction of the classified UPDRS subscores for the 50th-minute compared with baseline of the second arm study groups.

\begin{tabular}{lccc}
\hline & \multicolumn{2}{c}{$\boldsymbol{p}$-Values - Baseline vs. Post-stim } \\
\cline { 2 - 4 } Subgroup & Active & Dry needling & Sham \\
\hline Tremor & $\mathbf{0 . 0 0 1}$ & 0.029 & 0.027 \\
Rigidity & $\mathbf{0 . 0 4 7}$ & 0.189 & 0.385 \\
Bradykinesia & $\mathbf{0 . 0 0 1}$ & 0.092 & 0.892 \\
Gait and postural instability & $\mathbf{< . 0 0 1}$ & 0.028 & 0.315 \\
Bulbar abnormalities & $\mathbf{0 . 0 0 1}$ & 0.551 & 0.635
\end{tabular}

t-test; $p<0.05$, Bonferroni correction $p<0.016$. Italic: significant in t-test, bold: significant after Bonferroni correction, bold and italic: significant in t-test and Bonferroni.

the active groups considering the clinically important difference (CID) values described by Shulman et al. ( $\triangle$ UPDRS 2.5, 5.2, and 10.8 for minimal, moderate, and large CIDs, respectively) indicated that the improvement in UPDRS scores obtained with electrostimulation of the IAMZ in the present study falls between a moderate and large CID (Shulman et al., 2010). In addition, dry needling of IAMZ group reached to a statistically significant improvement (but not a CID) in UPDRS scores which was not the case for the sham or placebo groups. The dry needling group results also underlined the significance of the IAMZ. Invasive deep brain stimulation modalities of neuromodulation utilize bilateral approaches to document UPDRS improvement rates. It must be noted that the results of the present study were obtained using only unilateral stimulation, and bilateral stimulation needs to be investigated.

In addition to UPDRS score improvements, the classified UPDRS subitem paired $t$-test analysis also demonstrated that symptomatic suppression for all subitem groups in both arms of the study, including tremor, rigidity, bradykinesia, gait and postural stability and bulbar abnormalities, were statistically significant for only the electrostimulation of IAMZ group. All of the other study groups were not statistically significant after $t$-test analysis for all of the subitem analysis.

The Bonferroni correction is a conservative approach that has advantages and disadvantages, especially for pilot studies. To clarify the outcomes of the conservative Bonferroni correction, we also performed analyses of the subscore groups. The suppression of tremor, rigidity, gait and postural stability and bradykinesia symptoms were still statistically significant after Bonferroni correction in the first arm of the study, and all subgroups (except rigidity) exhibited statistically significant reductions after bonferroni correction in the second arm of the study. In addition, although the improvements in bulbar abnormalities of the first arm and rigidity in the second arm were not statistically significant after the Bonferroni correction, there was a trend toward significance in both groups (p-crit: 0.025, $p$ : 0.030 for bulbar abnormalities in arm 1; and p-crit: $0.016, p: 0.047$ for rigidity in arm 2). Axial symptoms, including speech, have also been reported to worsen with DBS-STN (Tornqvist et al., 2005; Hammer et al., 2010; Moreau et al., 2011; Sidiropoulos et al., 2013). None of the subitems worsened in the active stimulation group in the present study, in contrast to other DBS-STN applications (Tornqvist et al., 2005; Hammer et al., 2010; Moreau et al., 2011; Sidiropoulos et al., 2013). On the other hand, DBSPPN has been reported to induce greater improvements in PIGD (Hamani et al., 2007; Tykocki et al., 2011). In the context of the different outcomes of DBS-STN and DBS-PPN on motor symptoms and the outcomes in the present study, we suggest that the underlying mechanism of IAMZ stimulation may be related to PPN-associated pathways rather than STN-associated pathways or that both the STN and the PPN may be responsible for the observed effect; however, a sole contribution of the STN is not suggested.

The results of the bilateral assessments of the symptomatic improvements of $\mathrm{PD}$ patients before and after electrical stimulation of the IAMZ revealed that unilateral stimulation (ipsilateral to dominant, lateralized motor symptoms) of the IAMZ relieved PD symptoms on both sides of patients in both arms of this study. These improved contralateral motor symptoms may suggest a bihemispheric contribution of the intrinsic auricular muscles. Whether the stimulation of facial nerve branches over the intrinsic auricular muscles is solely responsible for all of the bilateral symptomatic improvements observed in this study or whether other nerves in the IAMZ also contribute remains unclear. The C2 spinal nerve may also play a role in bilateral symptomatic improvement. Although the C2-related role of the propriospinal tracts is more active on the ipsilateral side, animal studies demonstrated that $\mathrm{C} 2$ spinal nerves have ipsilateral and contralateral connections with the interpositus nucleus of the cerebellum (Matsushita and Xiong, 2001). As such, C2 spinal nerve stimulation may act on the bilateral PPN through bilateral cerebellar nuclei, and bilateral PPN stimulation may act on the bilateral reticulospinal tract to modulate bilateral motor symptoms. However, C2 zone stimulation in the sham group of the second arm was ineffective.

Autonomic nerve modulation may also contribute to the bilateral symptomatic relief observed in the present study. Lowfrequency vagal nerve stimulation leads to impaired vagus nerve function and inhibition of the dopamine system in brain structures (Ziomber et al., 2012). Considering that low- and high-frequency electrostimulation have different outcomes on the autonomic nerve system and on neurotransmitters (Cakmak et al., 2008, 2016; Zhao, 2008), high-frequency stimulation of the Arnold branch of the vagus nerve may in fact have the opposite effect. The present study used $130 \mathrm{~Hz}$ stimulation, which is very high compared with the frequency $(0.5 \mathrm{~Hz})$ that inhibits the dopaminergic system via vagus nerve stimulation (Ziomber et al., 2012). Therefore, the beneficial bilateral effects obtained in the present study may also be derived from improved dopamine levels. Although there was not a formal feedback survey, the patients in the active group also reported that they felt like they had their levodopa pills. The underlying neurotransmitter related mechanism of action will be the focus of animal studies in addition to levodopa integrated clinical trials for the IAMZ stimulation.

A recent study reported bilateral $\mathrm{c}$-fos activation in the nucleus tractus solitarius (NTS) and the locus coerulei (LC) after stimulation of the left cavum concha area, which is located in the bed of the Arnold branch of the vagus nerve 
in rats (Ay et al., 2015) (Figures 1A,B). On the other hand, transganglionic neurotracing studies with horseradish peroxidase (HRP) injections to the central cut end of the Arnold branch did not result in LC labeling (Nomura and Mizuno, 1984). Interestingly, the same study investigated left cavum concha stimulation and reported c-fos activation in the facial nerve nucleus in one of the experimental animals. The cavum concha is located next to the helicis minor muscle, and facial nerve fibers to the helicis minor may also be distributed in this area. Therefore, it is always possible that some facial nerve fibers will be stimulated along with the Arnold branch of the vagus nerve within the cavum concha. The authors attributed the c-fos labeling in the bilateral LC to projections from the NTS and as a result of stimulation of the Arnold branch of the vagus nerve. The Arnold branch of the vagus-NTS-LC axis may not be the only option for bilateral LC c-fos labeling after cavum concha stimulation, as sympathetic nerves also contribute to the cavum concha area within the arterial walls, and the cervical sympathetic ganglia were not investigated for possible labeling in that particular study. A major input to the LC originates from the nucleus paragigantocellularis (nPGi) in the reticular formation (Ennis and Aston-Jones, 1988; Kessel et al., 2008) (Figure 1B). The nPGi directly innervates sympathetic neurons and receives inputs (Ennis and Aston-Jones, 1988; Aston-Jones et al., 1996; Berntson et al., 1998) (Figures 1A,B). This pathway is involved in transmitting information of sympathetic control and state between the nPGi and the LC (Garcia-Rill, 1991; Limousin et al., 1997) (Figures 1A,B). In addition, the nPGi has been shown to be antidromically activated by electrostimulation of the LC, which may also be the case for sympathetic afferents. The major efferent pathways of the LC, the major wakefulnesspromoting nucleus, project to the PPN (Samuels and Szabadi, 2008) (Figure 1B). Therefore, the possible role of the auricular sympathetic nerves in LC activation and, as a consequence, their possible effects on motor regulation via the LC-PPN axis, cannot be excluded. In contrast, the sham stimulation zone, which included the $\mathrm{C} 2$ and sympathetic nerves in the second arm of the study, did not affect the UPDRS motor scores, which may suggest that the sympathetic nerves had a minimal role in the UPDRS improvements observed in the present study. On the other hand, the distribution of the sympathetic nerves may have less contribution to sham zone stimulation area in comparison to the IAMZ electrostimulation zones so that sympathetic nerves may still have a potential role in the observed clinical improvement.

Although there are different possible anatomical pathways from the IAMZ to the LC-PPN axis that need to be investigated, the demonstration of bilateral LC activation through unilateral stimulation of the cavum concha area (Ay et al., 2015) may also explain the bilateral motor improvements observed in our study.

Proprioception nerve fibers of the intrinsic auricular muscles may project to the mesencephalic trigeminal nucleus, to the cuneate nucleus, or both. The mesencephalic trigeminal nucleus projects to the reticular formation and to the spinal cord (Usunoff et al., 1997) (Figures 1A,B). This connection indicates the presence of potential intrinsic auricular muscle proprioception feedback over reticular formation, which is the PPN's gateway for influencing movement and posture. On the other hand, imaging studies have revealed that the cortical representation of the ear is distributed not as a single zone over the cortical face-head representation area but as numerous different zones over the face, head and neck representation areas (Nihashi et al., 2001). If this unique auricular sensory representation finding is extrapolated to intrinsic auricular muscle representations, then the proprioceptive feedback of the intrinsic auricular muscles may also contribute to the neck proprioceptive center, the nucleus cuneatus. A recent study also supported such a connection: in an fMRI study, stimulation of the antitragicus muscle zone as a control demonstrated activation of the nucleus cuneatus, whereas there was no activation in the nucleus cuneatus when a nonmuscular area of the ear was stimulated (Frangos et al., 2015) (Figures 1A,B). The nucleus cuneatus and the PPN are the two major components of the mesencephalic locomotor region that modulates PGID (Garcia-Rill, 1991). The SCM, where the transcutaneous electrode was placed, may also play a role in nucleus cuneatus modulation because of the alternating anodecathode stimulation method that we used in the present study. On the other hand, the effect would be relatively small compared with the effect that occurs by IAMZ stimulation because of the transcutaneous electrode usage and the very low power of the stimulus. We did not observe any contractions of the SCM during stimulation. In addition, the patients also reported a distinct tingling sensation over the IAMZ but not of the SCM where the transcutaneous electrode was placed. The different surface size of the needle electrodes placed on the IAMZ compared with the transcutaneous electrodes on the SCM may be the reason behind this difference, in addition to the skin conductance factor between the needle electrodes and the transcutaneous electrodes. In conclusion, the contribution of the SCM electrode to the symptomatic improvements observed in the present study, if any, would be minimal. The sham region in the present study is selected to eliminate the potential effect of stimulation of only the C2/sympathetic nerve by ensuring no or minimal stimulation of the SCM, the vagus facial nerve and the trigeminal nerve, on the other hand the character of the sham design has limitations for surface SCM stimulation contributions (if any). Different sham group designs are needed to eliminate the SCM only stimulation effects.

The upper face muscles, such as the orbicularis oculi and frontalis muscles, have similar bilateral hemispheric connections; however, reports of the muscle spindle content of the orbicularis oculi highlight the lack of muscle spindles in this muscle group (Urban et al., 2004). In addition, these muscles are actively used to as mimics and as sphincters, and the contraction of these muscles with external electrostimulation may induce visible contractions that counteract their functions. In conclusion, these muscles are not the ideal targets, not only because they lack muscle spindles but also because they are actively used in daily life, and therefore, the contraction of these muscles may be annoying to patients. Subthreshold stimulation that does not induce visible contractions of these muscles may be another option for an alternative non-invasive electrostimulation modality for modulating similar networks related to IAMZ, but the lack of muscle spindles is still a major concern. On the other hand, intrinsic auricular muscles have active roles in shaping 
the external ear surface features during the prenatal period and become functionally regressed but anatomically intact in adult humans, such that stimulation of intrinsic muscles does not induce the visible contractions observed in the frontalis and orbicularis oculi (Zerin et al., 1982; Yotsuyanagi et al., 1999). Finally, although no studies on the muscle spindle content of human intrinsic auricular muscles have been published thus far, muscle spindles have been examined in peri-auricular muscles in non-human primates, including, rhesus monkeys (Lovell et al., 1977). The statistically significant (but clinically not significant) effect that was observed in the IAMZ dry needling group in the second arm of the study also underlines the significance of the IAMZ zone during minimal stimulation. In addition, it is worth noting that electrostimulation with the same parameters of a muscle-free area of the auricula in the sham group had no effect on UPDRS scores.

The current study was a pilot study performed on a small group of patients that investigated the immediate effects of IAMZ stimulation on PD motor symptoms. Although the study includes numerous groups including sham, placebo, dry needling and active, the SCM contribution (if any), and the potential perception differences of the sham and real IAMZ stimulations were the limitations. The results require further validation in subsequent studies with more participants. Further investigations of bilateral stimulation applications and different stimulation frequencies are also needed to clarify the efficacy of the technique, as in DBS, for patient-specific frequency effects on tremor. In addition, although postural tremor and tremor at rest significantly improved after active stimulation, we observed that tremor developed more erratically and was modulated by the emotional states of patients. In addition, the patients also well-tolerated the device without any side effect or discomfort with short term $(20 \mathrm{~min})$ stimulation. The usability of the device for longer terms will be the focus of future trials. Responsiveness regarding the stage of the disease, different

\section{REFERENCES}

Alstermark, B., Isa, T., Lundberg, A., Pettersson, L. G., and Tantisira, B. (1989). The effect of low pyramidal lesions on forelimb movements in the cat. Neurosci. Res. 7, 71-75. doi: 10.1016/0168-0102(89)90038-2

Alstermark, B., Lundberg, A., Norrsell, U., and Sybirska, E. (1981). Integration in descending motor pathways controlling the forelimb in the cat. 9. Differential behavioural defects after spinal cord lesions interrupting defined pathways from higher centres to motoneurones. Exp. Brain Res. 42, 299-318. doi: 10.1007/ BF00237496

Alvord, L. S., and Farmer, L. B. (1997). Anatomy and orientation of the human external ear. J. Am. Acad. Audiol. 8, 383-390.

Aston-Jones, G., Rajkowski, J., Kubiak, P., Valentino, R. J., and Shipley, M. T. (1996). Role of the locus coeruleus in emotional activation. Prog. Brain Res. 107, 379-402. doi: 10.1016/S0079-6123(08)61877-4

Ay, I., Napadow, V., and Ay, H. (2015). Electrical stimulation of the vagus nerve dermatome in the external ear is protective in rat cerebral ischemia. Brain Stimul. 8, 7-12. doi: 10.1016/j.brs.2014.09.009

Berntson, G. G., Sarter, M., and Cacioppo, J. T. (1998). Anxiety and cardiovascular reactivity: the basal forebrain cholinergic link. Behav. Brain Res. 94, 225-248. doi: 10.1016/S0166-4328(98)00041-2

Braak, H., del Tredici, K., Rub, U., de Vos, R. A., Jansen Steur, E. N., and Braak, E. (2003). Staging of brain pathology related to sporadic Parkinson's disease. Neurobiol. Aging 24, 197-211. doi: 10.1016/S0197-4580(02)00065-9 stimulation frequencies and long-term effects should also be investigated. Moreover, we only observed the short-term effects of stimulation, and the second arm of the study indicates that the effect may persist up to the 30th-minute after termination of stimulation, which corresponded to the 50th-minute after the initiation of the stimulation. Prolonged efficacy will also be the focus of the next phase of clinical trials. Responsiveness regarding the stage of the disease must also be investigated in future studies.

\section{CONCLUSION}

We provide the first demonstration of a potential role of IAMZ electrical stimulation in improving (moderate to large clinical improvement) the clinical motor symptoms of $\mathrm{PD}$ patients in the short term. The underlying neuronal pathways and mechanisms need to be investigated in future studies.

\section{AUTHOR CONTRIBUTIONS}

Developed the concept: YC; experiment design: YC, YO, SE; performed stimulation experiments: $\mathrm{YC}, \mathrm{BO}$; performed patient enrollments and/or clinical assessments: HA, GK, SE, AG, OC; development of hardware: $\mathrm{HU}, \mathrm{SO}, \mathrm{YC}$; development of software and code writing: $\mathrm{HU}, \mathrm{SO}, \mathrm{BO}, \mathrm{YC}$; development of analytical tools: $\mathrm{HU}, \mathrm{SO}, \mathrm{BO}, \mathrm{YC}$; data collection: $\mathrm{BO}, \mathrm{YC}, \mathrm{OC}, \mathrm{SO}$; analyzed data: YC, SE, AG, BO, YO, SE; wrote the draft: YC; wrote the main paper: YC, HA, GK, SE, AG, BO, OC, YO, HU, BO.

\section{FUNDING}

This project was supported by Koç University, School of Medicine.

Burn, D. (2013). Oxford Textbook of Movement Disorders. Oxford: Oxford University Press, 16-22. doi: 10.1093/med/9780199609536.001.0001

Cakmak, Y. O., Akpinar, I. N., Ekinci, G., and Bekiroglu, N. (2008). Pointand frequency-specific response of the testicular artery to abdominal electroacupuncture in humans. Fertil. Steril. 90, 1732-1738. doi: 10.1016/j. fertnstert.2007.08.013

Cakmak, Y. O., Akpinar, I. N., and Yoldemir, T. (2016). Decreasing the uterine blood flow with electroacupuncture: bidermatomal and monodermatomal applications. Gynecol. Obstet. Invest. 82, 151-156. doi: 10.1159/00044 6947

Costa, J., Valls-Sole, J., Valldeoriola, F., Rumia, J., and Tolosa, E. (2007). Motor responses of muscles supplied by cranial nerves to subthalamic nucleus deep brain stimuli. Brain 130, 245-255. doi: 10.1093/brain/awl336

Daniel, E. E., and Paton, D. M. (1975). Methods in Pharmacology, Smooth Muscle, Vol. 3. New York, NY: Plenum Press, 442. doi: 10.1007/978-1-46842751-6

Drake, R. L., Vogl, W., and Mitchell, A. W. M. (2015). Gray's Anatomy for Students. Philadelphia, PA: Churchill Livingstone, 954.

Drew, T. (1991). Functional organization within the medullary reticular formation of the intact unanesthetized cat. III. Microstimulation during locomotion. J. Neurophysiol. 66, 919-938.

Drew, T., and Rossignol, S. (1990). Functional organization within the medullary reticular formation of intact unanesthetized cat. I. movements evoked by microstimulation. J. Neurophysiol. 64, 767-781. 
Ennis, M., and Aston-Jones, G. (1988). Activation of locus coeruleus from nucleus paragigantocellularis: a new excitatory amino acid pathway in brain. J. Neurosci. 8, 3644-3657.

Frangos, E., Ellrich, J., and Komisaruk, B. R. (2015). Non-invasive access to the vagus nerve central projections via electrostimulation of the external ear: fMRI evidence in humans. Brain Stimul. 8, 624-636. doi: 10.1016/j.brs.2014.11.018

Fujita, T. (1934). Die motorische innervation der genuinen aurikularmuskeln beim menschen, nebst bemerkungen zu ihrer phylogenetischen herkunft. Anat. Anz. 78,321 .

Garcia-Rill, E. (1991). The pedunculopontine nucleus. Prog. Neurobiol. 36, 363-389. doi: 10.1016/0301-0082(91)90016-T

Gorska, T., and Sybirska, E. (1980). Effects of pyramidal lesions on forelimb movements in the cat. Acta Neurobiol. Exp. 40, 843-859.

Hamani, C., Stone, S., Laxton, A., and Lozano, A. M. (2007). The pedunculopontine nucleus and movement disorders: anatomy and the role for deep brain stimulation. Parkinsonism Relat. Disord. 13, S276-S280. doi: 10.1016/S13538020(08)70016-6

Hammer, M. J., Barlow, S. M., Lyons, K. E., and Pahwa, R. (2010). Subthalamic nucleus deep brain stimulation changes speech respiratory and laryngeal control in Parkinson's disease. J. Neurol. 257, 1692-1702. doi: 10.1007/s00415010-5605-5

Hanajima, R., Chen, R., Ashby, P., Lozano, A. M., Hutchison, W. D., Davis, K. D., et al. (2004). Very fast oscillations evoked by median nerve stimulation in the human thalamus and subthalamic nucleus. J. Neurophysiol. 92, 3171-3182. doi: $10.1152 /$ jn.00363.2004

Harting, J. K. (1997). The Global Cerebellum '97. Ph.D. thesis, University of Wisconsin Medical School, Madison, WI.

Hazrati, L. N., and Parent, A. (1992). Projection from the deep cerebellar nuclei to the pedunculopontine nucleus in the squirrel monkey. Brain Res. 585, 267-271. doi: 10.1016/0006-8993(92)91216-2

Hirsch, E. C., Graybiel, A. M., Duyckaerts, C., and Javoy-Agid, F. (1987). Neuronal loss in the pedunculopontine tegmental nucleus in Parkinson's disease and in progressive supranuclear palsy. Proc. Natl. Acad. Sci. U.S.A. 84, 5976-5980. doi: 10.1073 /pnas.84.16.5976

Hobbs, S. F., Oh, U. T., Chandler, M. J., Fu, Q. G., Bolser, D. C., and Foreman, R. D. (1992). Evidence that $\mathrm{C} 1$ and $\mathrm{C} 2$ propriospinal neurons mediate the inhibitory effects of viscerosomatic spinal afferent input on primate spinothalamic tract neurons. J. Neurophysiol. 67, 852-860.

Hughes, A. J., Daniel, S. E., Kilford, L., and Lees, A. J. (1992). Accuracy of clinical diagnosis of idiopathic Parkinson's disease: a clinico-pathological study of 100 cases. J. Neurol. Neurosurg. Psychiatry 55, 181-184. doi: 10.1136/jnnp.55.3.181

Jankowska, E., and Edgley, S. A. (2006). How can corticospinal tract neurons contribute to ipsilateral movements? A question with implications for recovery of motor functions. Neuroscientist 12, 67-79. doi: 10.1177/107385840528 3392

Jankowska, E., Lundberg, A., and Stuart, D. (1983). Propriospinal control of interneurons in spinal reflex pathways from tendon organs in the cat. Brain Res. 261, 317-320. doi: 10.1016/0006-8993(83)90636-4

Kably, B., and Drew, T. (1998). Corticoreticular pathways in the cat. I. Projection patterns and collaterization. J. Neurophysiol. 80, 389-405.

Karachi, C., Grabli, D., Bernard, F. A., Tande, D., Wattiez, N., Belaid, H., et al. (2010). Cholinergic mesencephalic neurons are involved in gait and postural disorders in Parkinson's disease. J. Clin. Invest. 120, 2745-2754. doi: 10.1172/ JCI42642

Keizer, K., and Kuypers, H. G. (1984). Distribution of corticospinal neurons with collaterals to lower brain stem reticular formation in cat. Exp. Brain Res. 54, 107-120. doi: 10.1007/BF00235823

Keizer, K., and Kuypers, H. G. (1989). Distribution of corticospinal neurons with collaterals to the lower brain stem reticular formation in monkey (Macaca fascicularis). Exp. Brain Res. 74, 311-318. doi: 10.1007/BF00248864

Kessel, F., Rosenfield, P., and Anderson, N. (2008). Expanding the Boundaries of Health and Social Science: Case Studies in Interdisciplinary Innovation. New York, NY: Oxford University Press, 27.

Lambru, G., Miller, S., and Matharu, M. S. (2013). The red ear syndrome. J. Headache Pain 2:83. doi: 10.1186/1129-2377-14-83

Lavoie, B., and Parent, A. (1994). Pedunculopontine nucleus in the squirrel monkey: projections to the basal ganglia as revealed by anterograde tracttracing methods. J. Comp. Neurol. 344, 210-231. doi: 10.1002/cne.903440204
Lawrence, D. G., and Kuypers, H. G. (1968). The functional organization of the motor system in the monkey: I. The effects of bilateral pyramidal lesions. Brain 91, 1-14. doi: 10.1093/brain/91.1.1

Lee, D., Henriques, D. Y., Snider, J., Song, D., and Poizner, H. (2013). Reaching to proprioceptively defined targets in Parkinson's disease: effects of deep brain stimulation therapy. Neuroscience 244, 99-112. doi: 10.1016/j.neuroscience. 2013.04.009

Limousin, P., Greene, J., Pollak, P., Rothwell, J., Benabid, A. L., and Frackowiak, R. (1997). Changes in cerebral activity pattern due to subthalamic nucleus or internal pallidum stimulation in Parkinson's disease. Ann. Neurol. 42, 283-291. doi: 10.1002/ana.410420303

Limousin, P., Pollak, P., Benazzouz, A., Hoffmann, D., Le Bas, J. F., Broussolle, E., et al. (1995). Effect of parkinsonian signs and symptoms of bilateral subthalamic nucleus stimulation. Lancet 345, 91-95. doi: 10.1016/S0140-6736(95)90062-4

Little, S., and Brown, P. (2012). What brain signals are suitable for feedback control of deep brain stimulation in Parkinson's disease? Ann. N. Y. Acad. Sci. 1265, 9-24. doi: 10.1111/j.1749-6632.2012.06650.x

Lovell, M., Sutton, D., and Lindeman, R. C. (1977). Muscle spindles in nonhuman primate extrinsic auricular muscles. Anat. Rec. 189, 519-523. doi: 10.1002/ar. 1091890310

Luccarini, P., Gahery, Y., and Pompeiano, O. (1990). Cholinoceptive pontine reticular structures modify the postural adjustments during the limb movements induced by cortical stimulation. Arch. Ital. Biol. 128, 19-45.

Magni, F., and Willis, W. D. (1964). Cortical control of brain stem reticular neurons. Arch. Ital. Biol. 102, 418-433.

Massion, J. (1992). Movement, posture and equilibrium: interaction and coordination. Prog. Neurobiol. 38, 35-56. doi: 10.1016/0301-0082(92)90034-C

Matsuo, K., and Hirose, T. (1987). Tragicus and antitragicus muscles as constrictors of the external auditory meatus. Eur. J. Plastic Surg. 10, 82-83. doi: 10.1007/ BF00578381

Matsushita, M., and Xiong, G. (2001). Uncrossed and crossed projections from the upper cervical spinal cord to the cerebellar nuclei in the rat, studied by anterograde axonal tracing. J. Comp. Neurol. 432, 101-118. doi: 10.1002/cne. 1091

McFarland, N. R., and Haber, S. N. (2000). Convergent inputs from thalamic motor nuclei and frontal cortical areas to the dorsal striatum in the primate. J. Neurosci. 20, 3798-3813.

Mima, T., Sadato, N., Yazawa, S., Hanakawa, T., Fukuyama, H., Yonekura, Y., et al. (1999). Brain structures related to active and passive finger movements in man. Brain 122, 1989-1997. doi: 10.1093/brain/122.10.1989

Moreau, C., Pennel-Ployart, O., Pinto, S., Plachez, A., Annic, A., Viallet, F., et al. (2011). Modulation of dysarthropneumophonia by low-frequency STN DBS in advanced Parkinson's disease. Mov. Disord. 26, 659-663. doi: 10.1002/mds. 23538

Mori, S., Matsuyama, K., Kohyama, J., Kobayashi, Y., and Takakusaki, K. (1992). Neuronal constituents of postural and locomotor control systems and their interactions in cats. Brain Dev. 14, S109-S120.

Muthusamy, K. A., Aravamuthan, B. R., Kringelbach, M. L., Jenkinson, N., Voets, N. L., Johansen-Berg, H., et al. (2007). Connectivity of the human pedunculopontine nucleus region and diffusion tensor imaging in surgical targeting. J. Neurosurg. 107, 814-820. doi: 10.3171/JNS-07/10/0814

Nambu, A., Takada, M., Inase, M., and Tokuno, H. (1996). Dual somatotopical representations in the primate subthalamic nucleus: evidence for ordered but reversed body-map transformations from the primary motor cortex and the supplementary motor area. J. Neurosci. 16, 2671-2683.

Nambu, A., Tokuno, H., Hamada, I., Kita, H., Imanishi, M., Akazawa, T., et al. (2000). Excitatory cortical inputs to pallidal neurons via the subthalamic nucleus in the monkey. J. Neurophysiol. 84, 289-300.

Nambu, A., Tokuno, H., Inase, M., and Takada, M. (1997). Corticosubthalamic input zones from forelimb representations of the dorsal and ventral divisions of the premotor cortex in the macaque monkey: comparison with the input zones from the primary motor cortex and the supplementary motor area. Neurosci. Lett. 239, 13-16. doi: 10.1016/S0304-3940(97)00877-X

Nihashi, T., Kakigi, R., Kawakami, O., Hoshiyama, M., Itomi, K., Nakanishi, H., et al. (2001). Representation of the ear in human primary somatosensory cortex. Neuroimage 13, 295-304. doi: 10.1006/nimg.2000.0695

Nomura, S., and Mizuno, N. (1984). Central distribution of primary afferent fibers in the Arnold's nerve (the auricular branch of the vagus nerve): a 
transganglionic HRP study in the cat. Brain Res. 292, 199-205. doi: 10.1016/ 0006-8993(84)90756-X

Payoux, P., Remy, P., Damier, P., Miloudi, M., Loubinoux, I., Pidoux, B., et al. (2004). Subthalamic nucleus stimulation reduces abnormal motor cortical overactivity in Parkinson disease. Arch. Neurol. 61, 1307-1313. doi: 10.1001/ archneur.61.8.1307

Peuker, T. E., and Filler, T. J. (2002). The nerve supply of the human auricle. Clin. Anat. 15, 35-37. doi: 10.1002/ca.1089

Postuma, R. B., Gagnon, J. F., Vendette, M., Charland, K., and Montplaisir, J. (2008). Research paper REM sleep behaviour disorder in Parkinson's disease is associated with specific motor features. J. Neurol. Neurosurg. Psychiatry 79, 1117-1121. doi: 10.1136/jnnp.2008.149195

Samuels, E. R., and Szabadi, E. (2008). Functional neuroanatomy of the noradrenergic locus coeruleus: its roles in the regulation of arousal and autonomic function part I: principles of functional organisation. Curr. Neuropharmacol. 6, 235-253. doi: 10.2174/15701590878577 7229

Sasaki, S. (2004). Dexterous finger movements in primate without monosynaptic corticomotoneuronal excitation. J. Neurophysiol. 92, 3142-3147. doi: 10.1152/ jn.00342.2004

Schell, G. R., and Strick, P. L. (1984). The origin of thalamic inputs to the arcuate premotor and supplementary motor areas. J. Neurosci. 4, 539-560.

Schepens, B., and Drew, T. (2003). Strategies for the integration of posture and movement during reaching in the cat. J. Neurophysiol. 90, 3066-3086. doi: $10.1152 /$ jn. 00339.2003

Shulman, L. M., Gruber-Baldini, A. L., Anderson, K. E., Fishman, P. S., Reich, S. G., and Weiner, W. J. (2010). The clinically important difference on the unified Parkinson's disease rating scale. Arch. Neurol. 67, 64-70. doi: 10.1001/ archneurol.2009.295

Sidiropoulos, C., Walsh, R., Meaney, C., Poon, Y. Y., Fallis, M., and Moro, E. (2013). Low-frequency subthalamic nucleus deep brain stimulation for axial symptoms in advanced Parkinson's disease. J. Neurol. 260, 2306-2311. doi: 10.1007/s00415-013-6983-2

Takakusaki, K., Tomita, N., and Yano, M. (2008). Substrates for normal gait and pathophysiology of gait disturbances with respect to the basal ganglia dysfunction. J. Neurol. 255, 19-29. doi: 10.1007/s00415-0084004-7

Tattersall, T. L., Stratton, P. G., Coyne, T. J., Cook, R., Silberstein, P., Silburn, P. A., et al. (2014). Imagined gait modulates neuronal network dynamics in the human pedunculopontine nucleus. Nat. Neurosci. 17, 449-454. doi: 10.1038/nn. 3642

Tornqvist, A. L., Schalen, L., and Rehncrona, S. (2005). Effects of different electrical parameter settings on the intelligibility of speech in patients with Parkinson's disease treated with subthalamic deep brain stimulation. Mov. Disord. 20, 416-423. doi: $10.1002 / \mathrm{mds} .20348$
Tykocki, T., Mandat, T., and Nauman, P. (2011). Pedunculopontine nucleus deep brain stimulation in Parkinson's disease. Arch. Med. Sci. 7, 555-564. doi: 10. 5114/aoms.2011.24119

Urban, P., Bohl, J., Abrao, L., and Stofft, E. (2004). Absence of muscle spindles in human facial muscles. Klin. Neurophysiol. 35, 297.

Usunoff, K. G., Marani, E., and Schoen, J. H. R. (1997). The Trigeminal System in Man: Advances in Anatomy, Embryology and Cell Biology, Vol. 136. Berlin: Springer, 1-126. doi: 10.1007/978-3-642-60779-0

Wardman, D. L., Gandevia, S. C., and Colebatch, J. G. (2014). Cerebral, subcortical, and cerebellar activation evoked by selective stimulation of muscle and cutaneous afferents: an fMRI study. Physiol. Rep. 2:e00270. doi: 10.1002/phy 2.270

Whishaw, I. Q., Gorny, B., and Sarna, J. (1998). Paw and limb use in skilled and spontaneous reaching after pyramidal tract, red nucleus and combined lesions in the rat: behavioral and anatomical dissociations. Behav. Brain Res. 93, 167-183. doi: 10.1016/S0166-4328(97)00152-6

Yotsuyanagi, T., Nihei, Y., Shinmyo, Y., and Sawada, Y. (1999). Stahl's ear caused by an abnormal intrinsic auricular muscle. Plast. Reconstr. Surg. 103, 171-174. doi: 10.1097/00006534-199901000-00027

Zerin, M., Van Allen, M. I., and Smith, D. W. (1982). Intrinsic auricular muscles and auricular form. Pediatrics 69, 91-93.

Zhao, Z. Q. (2008). Neural mechanism underlying acupuncture analgesia. Prog. Neurobiol. 85, 355-375. doi: 10.1016/j.pneurobio.2008.05.004

Ziomber, A., Thor, P., Krygowska-Wajs, A., Zalecki, T., Moskala, M., Romanska, I., et al. (2012). Chronic impairment of the vagus nerve function leads to inhibition of dopamine but not serotonin neurons in rat brain structures. Pharmacol. Rep. 64, 1359-1367. doi: 10.1016/S1734-1140(12)70933-7

Zweig, R. M., Jankel, W. R., Hedreen, J. C., Mayeux, R., and Price, D. L. (1989). The pedunculopontine nucleus in Parkinson's disease. Ann. Neurol. 26, 41-46. doi: 10.1002/ana.410260106

Conflict of Interest Statement: YC, HU, SO, and BO have a related pending patent application.

The other authors declare that the research was conducted in the absence of any commercial or financial relationships that could be construed as a potential conflict of interest.

Copyright (c) 2017 Cakmak, Apaydin, Kiziltan, Gündüz, Ozsoy, Olcer, Urey, Cakmak, Ozdemir and Ertan. This is an open-access article distributed under the terms of the Creative Commons Attribution License (CC BY). The use, distribution or reproduction in other forums is permitted, provided the original author (s) or licensor are credited and that the original publication in this journal is cited, in accordance with accepted academic practice. No use, distribution or reproduction is permitted which does not comply with these terms. 